\title{
KU-MTL at SemEval-2018 Task 1: Multi-task Identification of Affect in Tweets
}

\author{
Thomas Nyegaard-Signori, Casper Veistrup Helms, \\ Johannes Bjerva, Isabelle Augenstein \\ Department of Computer Science \\ University of Copenhagen \\ $\{$ sfq340,wqx727\}@alumni.ku.dk, \{bjerva, augenstein\}@di.ku.dk
}

\begin{abstract}
We take a multi-task learning approach to the shared Task 1 at SemEval-2018. The general idea concerning the model structure is to use as little external data as possible in order to preserve the task relatedness and reduce complexity. We employ multi-task learning with hard parameter sharing to exploit the relatedness between sub-tasks. As a base model, we use a standard recurrent neural network for both the classification and regression subtasks. Our system ranks 32nd out of 48 participants with a Pearson score of 0.557 in the first subtask, and 20th out of 35 in the fifth subtask with an accuracy score of 0.464 .
\end{abstract}

\section{Introduction}

We consider the task of identifying affect in tweets, as described in Mohammad et al. (2018). Given a tweet, the task is to predict the emotions and their corresponding intensities which the tweet portrays. Previous approaches to this task are outlined in Mohammad and Bravo-Marquez (2017). The winning team of the SemEval EmoInt 2017, presented in Goel et al. (2017), tackled a similar task as the regression task presented in this year's SemEval Task 1. The winning system utilised an ensemble approach consisting of 5 sub-models and using a weighted average of these models to come up with the final result. This model is utilising most of the different approaches mentioned in the literature and combining them into one and with great success.

Our work bears resemblance to the runner up in the SemEval EmoInt 2017, Köper et al. (2017), who used a comparatively simple model consisting of a CNN-LSTM neural network. The difference between the models presented in this paper and the IMS system is the utilisation of lexicons, and that we take a multi-task learning approach.
We focus on two subtasks of this shared task, namely emotion intensity regression and emotion classification (Sub-tasks 1 and 5). These were chosen because of the overlap in tweets but differing truth labels value and types. Furthermore, we only consider the English versions of the subtasks.

\section{System Description}

Our system is a standard RNN, with the exception that we approach the task using multi-task learning via hard parameter sharing (Caruana, 1993). We will now present the details of our implementation.

\subsection{Preprocessing of Text and Textual Representation}

We use embedded word representations as input to our system, initialised to the weights from a large set of pre-trained embeddings which have been trained on Twitter data ( $\sim 400$ million tweets). These embeddings were obtained from Godin et al. (2015). Words which are out-of-vocabulary are replaced with UNK. Furthermore, user mentions and numbers get mapped to a place-holder instead of their actual values.

Since the model used both word and character representations, the characters are read in separately, although the same basic principle is followed. Every character is represented by an embedded representation, which is initialised randomly prior to training.

\subsection{Augmentation of Data}

Since the tweets have different formats of truth labels, one is a singular value (regression) and one is a multi-label list (classification), reading in the labels has to be augmented. Since the tweets are reused in the two tasks, some of the regression tweets can be augmented with their respective classification label, although, if no classification label is present, the truth labels are set to -1 , which 


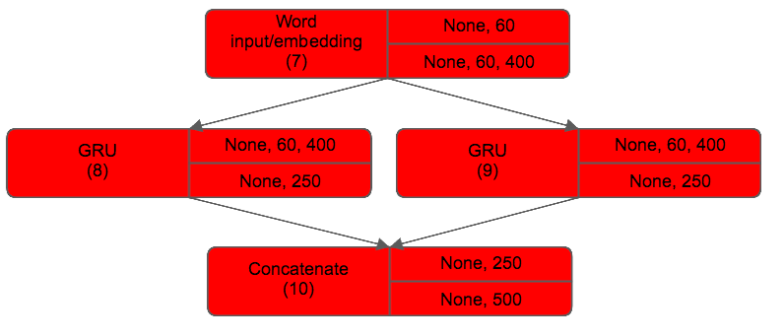

Figure 1: The word input part of the full model.

then acts as a mask. Since there are more regression tweets than classification, the augmentation is done this way around. All the tweets are padded differently based on whether or not the tweet is being read as a word representation or character representation, and tweets longer than the specified padding values are cut down to size.

\subsection{Word Input Model}

The word input to the model is $60 \cdot 400$ dimensional vectors that have been passed through the preprocessing and augmentation specified in section 2.1 and 2.2 ((7) in Figure 1). These vectors are then passed into two 250-dimensional, bidirectional GRUs which traverse the tweet front to back and vice versa, and the outputs of the two GRU layers are then concatenated ((8) and (9) in Figure 1). This output is then batch normalised and dropout is applied ((10) in Figure 1). This output of the word submodel is then concatenated later with the character submodel.

\subsection{Character Input Model}

The character input to the model is $256 \cdot 400$ dimensional vectors that have been through the same preprocessing and augmentation as the word inputs ((1) in Figure 2). These vectors then get passed into a residual neural network which works as a loop of batch normalisations, dropout applications and one-dimensional convolutions ((2) in Figure 2). Each loop ends with an addition of the values at the start of the loop and the current result and then a max pooling. These vectors are then passed into two GRU layers similar to the word input ((4) and (5) in Figure 2) which are then concatenated and passed along to be connected with the word input part ((6) in Figure 2).

\subsection{Full Model}

The combined model consists of four submodels, one for each regression emotion. The combined

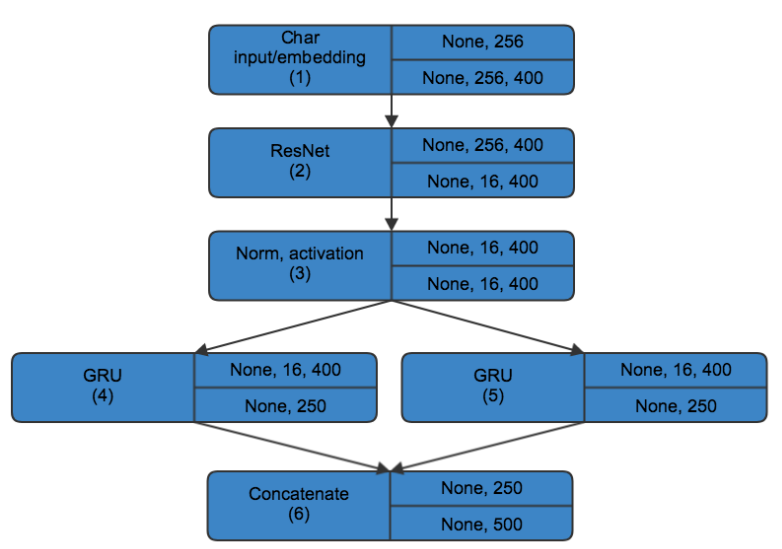

Figure 2: The character input part of the full model.

embeddings are used to generate classification labels. A high level overview can be seen in Figure 3 .

\subsection{Loss Functions}

Since the model is a multitask model, more than one loss function was needed. The model solves two tasks which can not share loss functions because of the inherent nature of the problem, one being a regression problem and the other a classification problem.

Regression loss function For the regression output of the model, mean squared error was chosen as a way to optimise the model with regards to Pearson-score. Since mean squared error seeks to minimise the difference between the prediction and the gold score, a low mean squared error will bring the Pearson score closer to one.

Classification loss function The loss function for the classification is a bit more convoluted since all regression tweets have regression labels, but not all regression tweets have classification labels. This is handled by way of a mask and the augmentation specified in section 2.2. Since the model has eleven output layers, there is a loss function for each of the eleven emotions/layers. The loss function ensures that tweets with no classification labels do not impact the updating of the weights of the model by giving the predicted values a loss of zero. The binary cross entropy loss function was modified to include a weighting parameter because of the uneven distribution of ones and zeros. The objective of the model is to identify the emotions indicated by a tweet, and as such a value of one is assigned a higher value. 


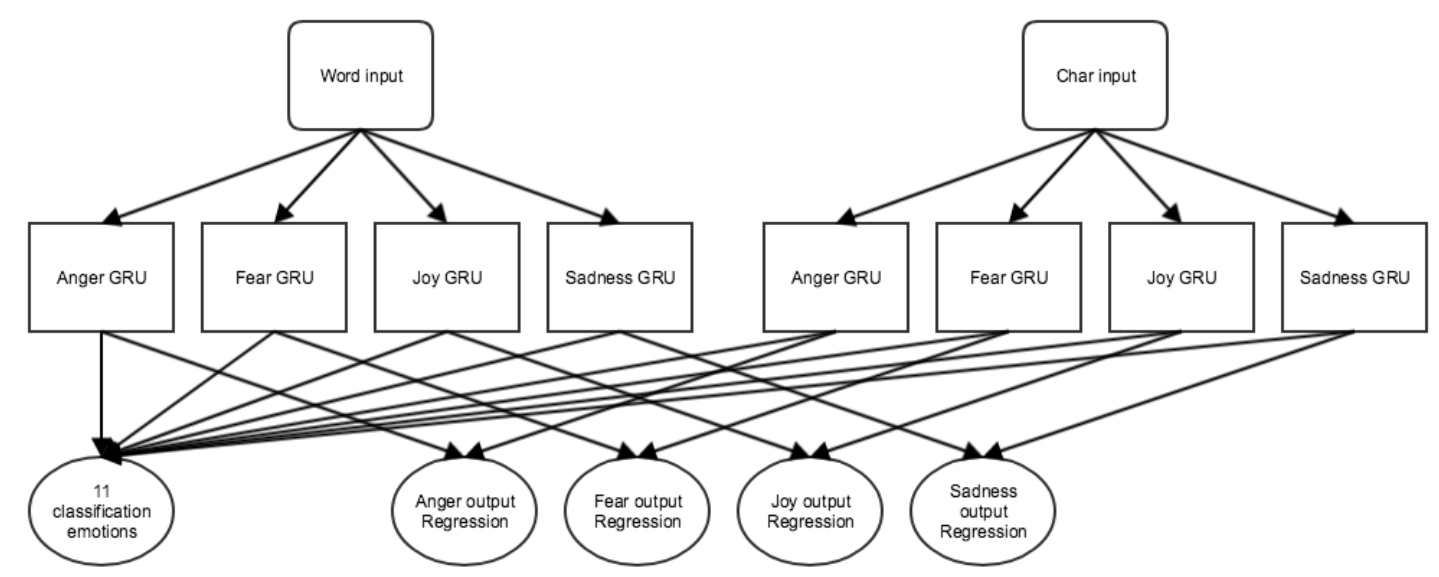

Figure 3: The character input part of the full model.

\section{Error Analysis}

\subsection{Regression Scores}

Since there is a considerable overlap in tweets, some tweets are reused in multiple emotions from Task 1 which then in turn can be reused a single time in Task 5. The actual numbers of unique and "duplicate" tweets are hard to resolve and presented a challenge in the first iterations of the model. Gold and predicted scores for an example instance are shown in Table 1 and 2.

\begin{tabular}{cccc}
\hline Anger score & Fear score & Joy score & Sadness score \\
\hline Gold : 0.517 & Gold : 0.800 & Gold : 0.197 & Gold : 0.707 \\
Pred : 0.449 & Pred : 0.953 & Pred : 0.139 & Pred : 0.756 \\
\hline
\end{tabular}

Table 1: Good prediction for regression task with the following tweet:

"we need to do something. something must be done!!!!!' your anxiety is amusing. nothing will be done. despair.".

\begin{tabular}{cccc}
\hline Anger score & Fear score & Joy score & Sadness score \\
\hline Gold : 0.953 & Gold : 0.621 & Gold : - & Gold : 0.680 \\
Pred : 0.620 & Pred : 0.346 & Pred : 0.430 & Pred : 0.326 \\
\hline
\end{tabular}

Table 2: Bad prediction for regression task with the following tweet:

"Don't fucking tag me in pictures as 'family first' when you cut me out 5 years ago. You're no one to me.”.

It is noticeable from the scoring shown in Table 1 and 2 that keywords such as 'familiy', 'anxiety' and 'fucking', for example, have a very large effect on the values predicted. These keyword cor- relations might have been better handled with the use of external data, such as emotive lexicons and the likes.

\subsection{Classification Scores}

Keeping in mind that the classification labels represent the emotions anger, anticipation, disgust, fear, joy, love, optimism, pessimism, sadness, surprise and trust, exemplary classification predictions are presented in Table 3 and Table 4.

\begin{tabular}{ccc}
\hline Predictions & Hit percentage \\
\hline Gold : 00100000100 & $82 \%$ \\
Pred : 01100000000 & \\
\hline
\end{tabular}

Table 3: Scores for classification task with the following tweet:

"Not sure tequila shots at my family birthday meal is up there with the best ideas I've ever had \#grim".

\begin{tabular}{ccc}
\hline Predictions & Hit percentage \\
\hline Gold : 01001110001 & $36 \%$ \\
Pred : 10100000000 & \\
\hline
\end{tabular}

Table 4: Bad prediction scores for classification task with the following tweet:

"SheenKL I assume the manga is \#good?".

There are certain structures that are prevalent in the correct and incorrect predictions. When looking at the amount of labels that are set in the datasets, it is evident just from the small amount of surprise or trust labels that have been set that these 
emotions will be harder to predict, since there are so few points of reference. Furthermore, when both anger and disgust labels are present in a tweet the model predicts better. This can be explained by the fact that there are a significant amount of tweets with these two labels.

\begin{tabular}{ccccc|c}
\hline Average score & Anger & Fear & Joy & Sadness & Classification \\
\hline 0.551 & 0.521 & 0.606 & 0.538 & 0.563 & 0.464 \\
\hline
\end{tabular}

Table 5: Overall scoring of the model, both regression and classification (Task 1 and 5).

Table 5 describes the overall scores from the model run on the test set in the evaluation period of the shared task.

\section{Related Work}

Multi-task Learning Neural networks make multi-task learning via (hard) parameter sharing particularly easy (Caruana, 1993) and has shown to be successful for a variety of NLP tasks, such as machine translation (Dong et al., 2015; Luong et al., 2016), keyphrase boundary classification (Augenstein and Søgaard, 2018), tagging (Martínez Alonso and Plank, 2017; Bjerva et al., 2016), complex word identification (Bingel and Bjerva, 2018), and natural language understanding (Augenstein et al., 2017). For sequence labelling, many combinations of tasks have been explored, e.g., by Martínez Alonso and Plank (2017); Bjerva $(2017 \mathrm{a}, \mathrm{b})$. An analysis of different task combinations was performed by Søgaard and Goldberg (2016); Bingel and Søgaard (2017). Ruder et al. (2017) presented a more flexible architecture, which learned what to share between the main and auxiliary tasks, and might require further investigation in future work. Augenstein et al. (2017) combine multi-task learning with semi-supervised learning for strongly related tasks with different output spaces. For this shared task, we opt for a simple hard parameter sharing strategy, though we would expect to see improvements with more involved architectures.

\section{Conclusion}

In this paper, we present our system for SemEval2018 Task 1 . We employed a simple multi-task architecture with hard parameter sharing to model Subtasks 1 and 5 jointly. The model achieved an average performance compared to the rest of the participants. We argue this is due to our not using external data or performing extensive additional engineering.

\section{Acknowledgments}

Isabelle Augenstein is supported by Eurostars grant Number E10138. We further gratefully acknowledge the support of NVIDIA Corporation with the donation of the Titan Xp GPU used for this research.

\section{References}

Isabelle Augenstein, Sebastian Ruder, and Anders Søgaard. 2017. Multi-task learning of keyphrase boundary detection. In Proceedings of $A C L$.

Isabelle Augenstein and Anders Søgaard. 2018. Multitask learning of pairwise sequence classification tasks over disparate label spaces. In Proceedings of NAACL, to appear.

Joachim Bingel and Johannes Bjerva. 2018. Crosslingual complex word identification with multitask learning. In Proceedings of Shared Task on CWI at BEA18.

Joachim Bingel and Anders Søgaard. 2017. Identifying beneficial task relations for multi-task learning in deep neural networks. In EACL. pages 164-169.

Johannes Bjerva. 2017a. One Model to Rule them all - Multitask and Multilingual Modelling for Lexical Analysis. Ph.D. thesis, University of Groningen.

Johannes Bjerva. 2017b. Will my auxiliary tagging task help? Estimating Auxiliary Tasks Effectivity in Multi-Task Learning. In Proceedings of NoDaLiDa. Linköping University Electronic Press, 131, pages 216-220.

Johannes Bjerva, Barbara Plank, and Johan Bos. 2016. Semantic tagging with deep residual networks. In Proceedings of COLING 2016. pages 3531-3541.

Richard A Caruana. 1993. Multitask connectionist learning. In In Proceedings of the 1993 Connectionist Models Summer School.

Daxiang Dong, Hua Wu, Wei He, Dianhai Yu, and Haifeng Wang. 2015. Multi-Task Learning for Multiple Language Translation. In Proceedings of ACL.

Fréderic Godin, Baptist Vandersmissen, Wesley De Neve, and Rik Van de Walle. 2015. Multimedia Lab @ ACL W-NUT NER Shared Task: Named Entity Recognition for Twitter Microposts using Distributed Word Representations .

Pranav Goel, Devang Kulshreshtha, Prayas Jain, and K.K. Shukla. 2017. Prayas at emoint 2017: An ensemble of deep neural architectures for emotion intensity prediction in tweets . 
Maximilian Köper, Evgeny Kim, and Roman Klinger. 2017. Ims at emoint-2017: Emotion intensity prediction with affective norms, automatically extended resources and deep learning .

Minh-Thang Luong, Quoc V. Le, Ilya Sutskever, Oriol Vinyals, and Lukasz Kaiser. 2016. Multi-task Sequence to Sequence Learning. In Proceedings of ICLR.

Héctor Martínez Alonso and Barbara Plank. 2017. When is multitask learning effective? Semantic sequence prediction under varying data conditions. In EACL. pages 44-53.

Saif M. Mohammad and Felipe Bravo-Marquez. 2017. Wassa-2017 shared task on emotion intensity .

Saif M. Mohammad, Felipe Bravo-Marquez, Mohammad Salameh, and Svetlana Kiritchenko. 2018. Semeval-2018 Task 1: Affect in tweets. In Proceedings of International Workshop on Semantic Evaluation (SemEval-2018). New Orleans, LA, USA.

Sebastian Ruder, Joachim Bingel, Isabelle Augenstein, and Anders Søgaard. 2017. Sluice networks: Learning what to share between loosely related tasks. arXiv preprint arXiv:1705.08142 .

Anders Søgaard and Yoav Goldberg. 2016. Deep multi-task learning with low level tasks supervised at lower layers. In $A C L$. volume 2, pages 231-235. 